\title{
Epidemiological Analysis of Symptomatic Gonococcal Infection among Male Patients attending STD Clinic
}

\author{
Dr. R. Snekavalli M. D. DVL ${ }^{1}$, Dr. S. Kalaivani M. D., DV ${ }^{2}$ \\ ${ }^{1}$ Assistant Professor of STD, Institute of Venereology, Madras Medical College, Chennai -600003, Tamil Nadu, India \\ Professor of STD, Institute of Venereology, Madras Medical College, Chennai -600003, Tamil Nadu, India
}

\begin{abstract}
Introduction: Gonorrhoea caused by Neisseria gonorrhoeae is one of the oldest known bacterial sexually transmitted infection that continues to cause a significant morbidity among the sexually active individuals. Aim: To study the epidemiological pattern of gonococcal infection in male patients with urethral discharge. Materials and Methods: A Retrospective study was conducted in 628 symptomatic male patients who attended Institute of Venereology, Madras Medical College with urethral discharge. Study period was from August 2011 - August 2016. Gram stain and culture was done from urethral discharge. Results: Among 628 cases, gonococci was positive by gram smear in 41 cases and by culture in 43 cases.Majority of them were in age group 20-25 years. Incubation period was 3-5 days in $51 \% .90 \%$ were unmarried. 26 were homosexual. Conclusion: To conclude gonococcal infection is most prevalent in unmarried males in the age group of 20-25 years with homosexual behaviour.
\end{abstract}

Keywords: Urethritis, Culture, Gram Stain, Homosexuals, Gonorrhoea

\section{Introduction}

Gonorrhoea is one of the oldest known bacterial sexually transmitted infection that continues to cause a significant morbidity among the sexually active individuals.

\section{Aim}

To study the epidemiological pattern of gonococcal infection in male patients with urethral discharge.

\section{Materials and Methods}

A Retrospective study was conducted in 628 symptomatic male patients who attended Institute of Venereology, Madras Medical College with urethral discharge. Study period was from August 2011 - August 2016.
Gram stain was done from urethral discharge and gonococc was identified by microscopy as gram negative bean shaped intracellular diplococci. It was followed by culture in chocolate agar and Modified Thayer Martin medium.Rectal and Pharyngeal swabs were taken from all cases who had history of oral or anal sex. Antibiotic sensitivity test by disc diffusion method was performed.

\section{Results}

Among 628 cases, gonococci was positive by gram smear in 41 cases and by culture in 43 cases.Majority of the males were in the age group 20-25 with a mean age of 26 years. $41 \%$ have completed $12^{\text {th }}$ standard, followed by $23 \%$ who were uneducated.

Majority of them (64.3\%) earned 3000-5000 per month.

Incubation period was 3-5 days in 51\% and 6-10 days in 23\%.(Fig :1)

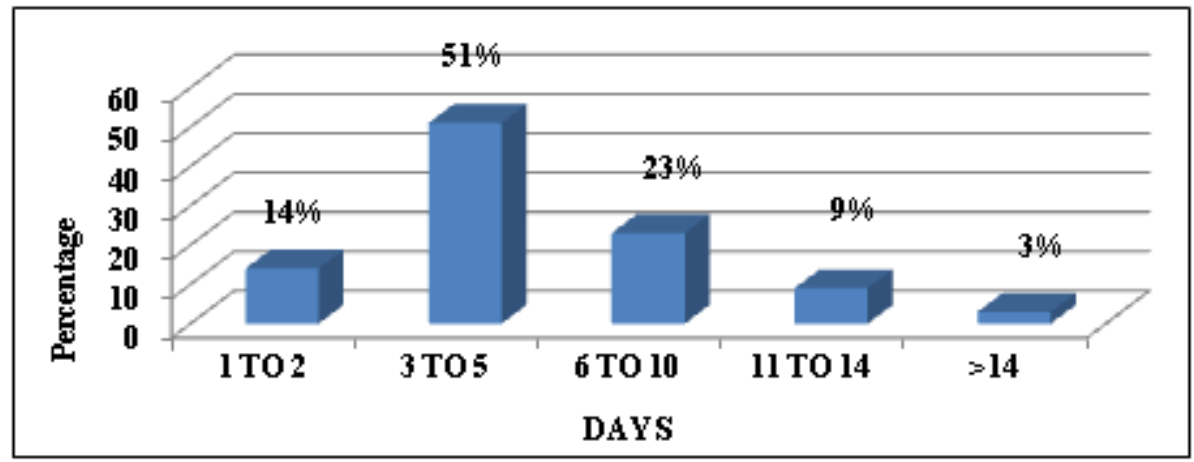

Figure 1: Incubation Period for Uretheral Discharge (Average 5.25 Days)

Among the study population, $90 \%$ were single and unmarried $.10 \%$ were married. $60 \%$ were homosexual, $28 \%$ were heterosexual followed by $12 \%$ bisexual.(Fig :2) 


\section{International Journal of Science and Research (IJSR) \\ ISSN (Online): 2319-7064 \\ Index Copernicus Value (2015): 78.96 | Impact Factor (2015): 6.391}

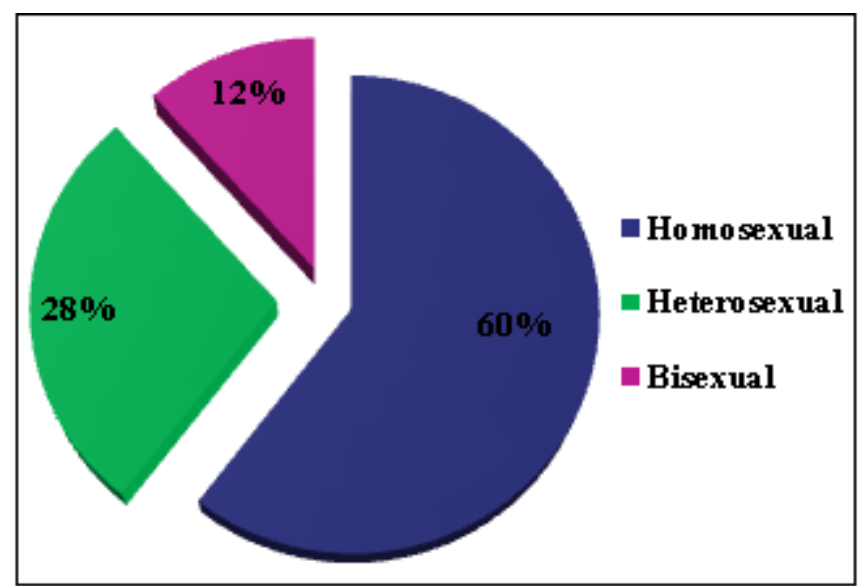

Figure 2: Sexual Behaviour

$32(74 \%)$ did not use condom for oral or anal sex and 11 (24\%) used condom sometimes.

$1(2 \%)$ case had pharyngeal gonorrhoea and $1(2 \%)$ had rectal gonorrhoea.

$3(43 \%)$ cases had genital warts ,2 cases(29\%) had syphilis and 1 case $(14 \%)$ had HIV , 1 case $(14 \%)$ had genital herpes. (Fig: 3)

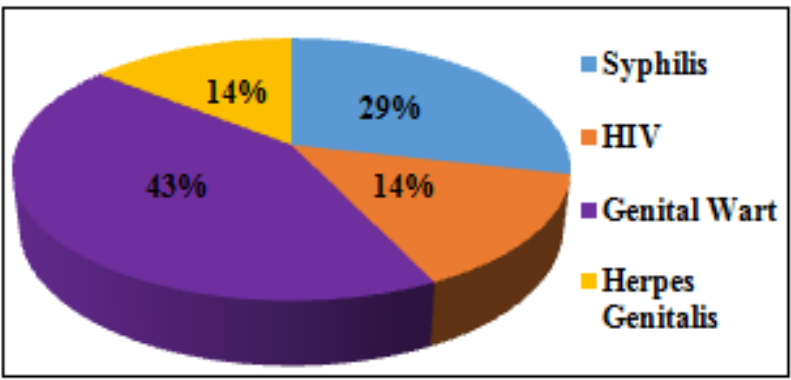

Figure 3: Associated STI'S

$41(95 \%)$ were treated with grey kit which consists of Tablet. Cefixime (400 mg)and Tablet. Azithromycin (1gram) and 2 cases of rectal and pharyngeal gonorrhoea (5\%) required Injection. Ceftriaxone $500 \mathrm{mg}$ IM stat according to NACO guidelines and symptoms resolved.

Of the 26 homosexual men positive for Gonococci, contact tracing was possible in $11(40 \%)$ of males only and they were treated with grey kit. Of the 17 heterosexual males, treatment was given to 4 spouses . Only 2 causal female contacts could be traced and treated. All cases were given grey kit in hand to give to their known partners who are unable to come to the OP.

\section{Discussion}

Men who have sex with men (MSM) are a vulnerable population for the spread of STI. MSM has bisexual behavior and are responsible for spread of STIs to males as well as females. Apart from being important for HIV transmission, they tend to have a different distribution pattern of STIs. ${ }^{[1]}$

Gonococci detected in cases of urethritis was $6.5 \%$ by microscopy which is lower than that reported by Ghosh et al ${ }^{[2]}$ in a urban STD centre in Kolkata which was $29 \%$. This could be because patients with acute gonorrhoea less frequently attend hospital clinic due to over the counter availability of effective antibiotics and widespread use of syndromic management. Although gonococcal culture is the gold standard, there are certain disadvantages of culturebased identification methods. These include the fastidiousness of the organism, which makes successful growth less likely if isolation media, specimen transport, and laboratory techniques, are not optimal ${ }^{[3]}$.

In Nucleic acid amplification test (NAAT) and multiplex PCR, self-collected genital specimens can be used. It can be used in detecting asymptomatic cases . NAATs offer greatly expanded sensitivities of detection, usually well above $90 \%$, while maintaining very high specificity, usually $\geq 99 \%{ }^{[4]}$

The most common age group in our study was 21-25 yrs( $62.7 \%$ ). This is slightly higher to a study conducted by Gupta et al ${ }^{[5]}$ at Pune in which most cases were in the age group 21-30 yrs. The average incubation period was 5.2 days with $51 \%$ developing uretheral discharge within 3-5 days and only $3 \%$ developed symptoms after 14 days. This is higher than the incubation period of 4 days reported by Gupta et al [5].

In our study, 39 (90\%) males were single, unmarried and 4 $(10 \%)$ were married. That gonorrhea infection was commonest in populations who had never married, and in the divorced population was reported by Bjekic et al [6]. $26(61 \%)$ were homosexual (MSM), 12 (27\%) were heterosexual, $6(12 \%)$ bisexual in our study population. This is similar to a study conducted by Garg et al ${ }^{[7]}$ in Suraksha clinc in 2012 in which and gonorrhoea was three times more common in MSM than in heterosexual.

Among men who reported anal sex, those reporting only insertive sex had lower incidence of gonorrhoea than did men who reported both insertive and receptive which was observed by Grov et $\mathrm{al}^{[\mathbf{8}] \cdot} 2 \%$ incidence of rectal and pharyngeal gonorrhoea was observed in our study, which is lower than that reported by Mathew et al ${ }^{[9]}$.

Rectal and pharyngeal swabs should always be taken in all MSMs as these sites harbour silent infection.

Contact tracing in males possible in $40 \%$ of cases which is lower than reported by Jayashree et al ${ }^{[10]}$ in which it was $50 \%$. Thus, the risk factors for gonococcal infection are being single, low socio-economic status, homosexual behaviour, more number of partners and no condom usage.

\section{Conclusion}

Combining NAAT test with gonococcal culture, will unearth more hidden cases of gonorrhoea. To conclude gonococcal infection is most prevalent in unmarried males in the age group of 20-25 years with homosexual behaviour. Men having sex with men form significant bridge population and hence targeted intervention and strategies focusing specifically on MSM for control of STDs and HIV are required. There is a critical need for increasing awareness about the correct condom use as well as more intensive efforts at contact tracing.

\section{Volume 6 Issue 1, January 2017




\section{References}

[1] Charu Nayyar, Ram Chander, Poonam Gupta, BL Sherwal. Evaluation of risk factors in patients attending STI clinic in a tertiary care hospital in North India. Indian J Sex Transm Dis Year:Year : 2015 | Volume : 36 | Issue : 1 | Page :48-52.

[2] Ghosh S. A study on the present scenario of STD management in an urban clinic in Kolkata. Indian $\mathrm{J}$ Dermatol Venereol Leprol 2002;68:82.

[3] Verma R, Seema Sood, Manjubala, Arti Kapil, VK Sharma. Diagnostic approach to gonorrhoea: Limitations. Indian J Sex Transm Dis Year : 2009 | Volume : 30 | Issue : 1 | Page : 61-64.

[4] Verma R, Sood S. Gonorrhoea diagnostics: An update. Indian J Med Microbiol 2016;34:139-45.

[5] Gupta C M, Sanghi S, Sayal S K, Das A L, Prasad G K. Clinical and bacteriological study of urethral discharge. Indian J Dermatol Venereol Leprol 2001;67:185-7.

[6] Bjekić M, Vlajinac H, Sipetić S.-Incidence of gonorrhea in Belgrade, 1988-1994 J Infect. 1998 Jul;37(1):44-7.

[7] Garg T, Chander R, Jain A, Barara M. Sexually transmitted diseases among men who have sex with men: A retrospective analysis from Suraksha clinic in a tertiary care hospital. Indian J Sex Transm Dis [serial online] 2012 [cited 2016 Nov 11];33:16-9.

[8] Grov C, Cain D, Rendina HJ, Ventuneac A, Parsons JT. Characteristics Associated With Urethral and Rectal Gonorrhea and Chlamydia Diagnoses in a US National Sample of Gay and Bisexual Men: Results From the One Thousand Strong Panel Sex Transm Dis. 2016 Mar;43(3):165-71.

[9] Matthew J. Mimiaga, SCD, MPH, ${ }^{*} \dagger$ Donna J. Helms, $\mathrm{MPH}, \ddagger$ L. Reisner, MA, ${ }^{*}$ Chris Grasso, MPH, ${ }^{*}$ Thomas Bertrand, MPH,§ Debra J. Mosure, PHD, Hillard Weinstock, MD, MPH, Catherine McLean, MD, and Kenneth H. Mayer, MD-Gonococcal, Chlamydia, and Syphilis Infection Positivity Among MSM Attending a Large Primary Care Clinic, Boston, 2003 to 2004.48-52.

[10] Jayasree P, Binitha MP, Najeeba R, Biju G. Clinical and epidemiological profile of sexually transmitted infections in a tertiary care centre in Kerala: A 1-year observational study. Indian J Dermatol Venereol Leprol 2015;81:500-3. 